\title{
Actualización de las Ecorregiones Terrestres de Perú propuestas en el Libro Rojo de Plantas Endémicas del Perú
}

\section{Update of the Terrestrial Ecoregions of Peru proposed in the Red Book of Endemic Plants of Peru}

\author{
BERNI BRITTO
}

Laboratorio de Biogeografía y Ecología Terrestres, Facultad de Ciencias Biológicas, Universidad Nacional Mayor de San Marcos - Ciudad Universitaria, Avenida Venezuela Cdra. 34 s/n, Lima 11, Apartado Postal 11-0058, Lima, Perú.

berni.britto@yahoo.com

\begin{abstract}
RESUMEN
En el 2007 se publicó el Libro Rojo de las Plantas Endémicas del Perú, donde se presentó una nueva propuesta de ecorregiones terrestres para el país. Las 13 ecorregiones propuestas no llegaron a ser descritas en su totalidad, ni representadas en un mapa. En este artículo se presenta la interpretación y actualización de las ecorregiones propuestas en el 2007, así como las modificaciones subsecuentes a las mismas, con base en los nuevos avances del conocimiento de la flora peruana y sudamericana, y los recursos informáticos disponibles actualmente. Las 15 nuevas ecorregiones propuestas son contrastadas con anteriores planteamientos de ecorregiones que han sido empleadas para describir el territorio peruano.
\end{abstract}

Palabras clave: Ecorregiones, límites altitudinales, Perú, flora, endemismo.

\begin{abstract}
In 2007, the Red Book of Endemic Plants of Peru was published, where a new proposal of terrestrial ecoregions of Peru was presented. The 13 proposed ecoregions were not fully described or represented on a map. This article presents the interpretation and update of the ecoregions proposed in 2007, as well as the subsequent modifications to these, based on new advances in knowledge of Peruvian and South American flora, as well as available information resources. The 15 new ecoregions are contrasted with those previously proposed and which have been used to describe the Peruvian territory.
\end{abstract}

KeYwords: Terrestrial ecoregions, Departmental and altitudinal limits, Peru, Flora, Endemism.

\section{INTRODUCCIÓN}

En el año 2006 se presentó el Libro Rojo de las Plantas Endémicas del Perú (León et al. 2007), el cual constituye hasta la fecha uno de los mayores esfuerzos en recopilación de especies de la flora peruana, después del catálogo de angiospermas y gimnospermas de Brako y Zarucchi (1993); en este libro se describió una modificación a las regiones ecológicas terrestres propuestas por Zamora para el Perú (Zamora 1996). Sin embargo, no se presentó un mapa con las modificaciones propuestas. El mapa que se presenta a continuación es la representación de las descripciones de ecorregiones del Libro Rojo de las Plantas Endémicas del Perú (en adelante, LRPEP), incluyendo algunas modificaciones en las áreas de las ecorregiones y las denominaciones de las mismas.

Las modificaciones propuestas se basan en las nuevas herramientas disponibles actualmente, como mapas de cobertura vegetal, avances del conocimiento de la flora peruana y sudamericana, así como los últimos estudios realizados para comprender los patrones de diversidad y evolución de la flora sudamericana.

\section{MATERIALES Y MÉTODOS}

Actualización de géneros endémicos del Perú Como parte de la información brindada para cada ecorregión propuesta en el LRPEP, se realizó una aproximación de la distribución de los géneros endémicos del Perú por cada ecorregión. Con la finalidad de proveer la misma información, se realizó una actualización de los mismos, para lo cual se utilizó bibliografía especializada (Andersson 1995, Andersson \& Taylor 1994, Leiva \& Zapata 2008, 
Porter \& Prather 2008, Panero 2009, Dillon \& Zapata 2010, Quispe \& Fuentes 2010, Gutiérrez 2010, Pruski 2012, Funk et al. 2013, Panero \& Freire 2013, León et al. 2013, Britto \& Arana 2014, Robinson \& Funk 2014, Saldivia \& Faúndez 2014) y bases de datos internacionales (Tropicos.org).

Los nombres propuestos para las ecorregiones actualizadas han sido tomados principalmente de las descritas en el LRPEP; en el caso de las modificaciones de nombres de algunas ecorregiones, así como las nuevas propuestas, se tomó en cuenta las denominaciones empleadas en diferentes estudios realizados sobre su flora y vegetación a nivel local, nacional y regional (ONERN 1976, Huber \& Rina 1997, Luteyn 1999, Linares-Palomino 2004, Sánchez-Vega \& Dillon 2006, Weigend et al. 2005, Britto \& Arana 2014).

DESCRIPCIÓN DE ECORREGIONES DEL LRPEP

En el LRPEP se presenta una nueva propuesta de ecorregiones del Perú en base a modificaciones a las ecorregiones propuestas por Zamora (1996). Esta nueva propuesta contempla 13 ecorregiones, las cuales son: Desierto Cálido Tropical (DCT): Localizado al norte del río Chicama. Desde el nivel del mar hasta 500 m s.n.m.; Desierto Semicálido Tropical (DST): Localizado a lo largo de la franja costera al sur del valle del Chicama. Desde el nivel del mar hasta 1.000 m s.n.m.; Matorral Desértico (MDE): Localizado mayormente en las laderas de la vertiente del Pacífico, desde 1.000 hasta 2.500 m s.n.m.; Meso-Andina (MA): Incluye las vertientes occidentales, las laderas de valles interandinos y el altiplano, entre 2.500 a 3.500 (-3.800) m s.n.m.; Puna Húmeda y Seca (PSH): Incluye la franja andina de Arequipa a La Libertad, entre 3.800 y 4.200 m s.n.m.; Puna Desértica (PD): Incluye la franja andina de Moquegua, Tacna y la parte occidental de Puno, entre 3.800 y 4.200 m s.n.m.; Páramo (PAR): Incluye la zona de Cajamarca y Piura, entre 3.500 y $4.200 \mathrm{~m}$; Altoandino (AA): incluye la zona sobre 4.200 m s.n.m.; Bosques Secos (BS): Incluye el bosque seco ecuatorial, entre 500 y $1.000 \mathrm{~m}$ en los departamentos de Tumbes, Piura y Lambayeque, así como las laderas bajas de los valles secos entre (500-)1.000 y 2.500 m s.n.m.; Bosques Pluviales Montanos (BPM): Localizados en la franja alta de la vertiente oriental y la faja entre los ríos Huancabamba y Chinchipe, entre 2.500 y 3.500 m s.n.m.; Bosques Muy Húmedos Montanos (BMHM): Por debajo de los bosques pluviales montanos en la vertiente oriental, entre $1.500 \mathrm{y}$ 2.500 m s.n.m.; Bosques Muy Húmedos Premontanos (BMHP): En la vertiente oriental. Entre 500 y $1.500 \mathrm{~m}$ s.n.m.; Bosques Húmedos Amazónicos (BHA): Incluye los bosques marcadamente no estacionales e inundables de Loreto, Madre de Dios y Ucayali así como departamentos aledaños, entre los 500 y los 100 m s.n.m.
REPRESENTACIÓN DE LAS ECORREGIONES LRPEP

En base a las descripciones de las ecorregiones se elaboró el mapa a través del programa ArcGIS 10.3, utilizando un modelo de elevación digital SRTM $90 \mathrm{~m}$ proporcionado por el Consortium for Spatial Information (CGIAR-CSI). Adicionalmente se comparó las áreas de las ecorregiones con el mapa de cobertura vegetal del Perú elaborado por el MINAM (2015) y observaciones realizadas en diferentes viajes de investigación en campo entre los años 2010 a 2016, con la finalidad de delimitar las ambigüedades presentes en la descripción de las ecorregiones en el LRPEP.

\section{RESULTADOS}

Actualización de GÉneros endémicos de Perú El LRPEP reconoció un total de 36 (37, sin considerar el género Weberbauerocereus Backeb.), en comparación a las 52 reconocidas por Brako \& Zarucchi (1993); esta reducción se dio por el incremento en el conocimiento de la distribución de las especies de plantas vasculares, así como reordenamientos nomenclaturales. La actualización de la lista de géneros endémicos dio como resultado el mismo número (37) (Tabla 1), por un recambio igualitario de cinco géneros nuevos (todos pertenecientes a la familia Asteraceae) y cinco géneros que dejaron de ser endémicos debido a cambios nomenclaturales (Chucoa Cabrera y Huthia Brand), registro de especies en otros países (Pseudonoseris H. Rob \& Brettell y Stilpnophyllum Hook. f.) y descripción de nuevas especies en otros países (Weberbauerella Ulbr. y Weberbauerocereus).

Una mención especial merece el género Weberbauerocereus, debido a que en el LRPEP no es considerado como un género endémico (aunque no se menciona que alguna de sus especies se registre en otro país), sin embargo, en la publicación "Todos los cactus del Perú" (Ostolaza 2014) se señala que este género es endémico al territorio peruano. No obstante, en el año 2010 se publica W. madidiensis Quispe \& A. Fuentes, una nueva especie del norte de Bolivia (Quispe \& Fuentes 2010).

Actualización de ecorregiones del Perú

Como resultado de la actualización de los géneros endémicos al territorio peruano y la revisión de los límites espaciales y altitudinales de las ecorregiones descritas en el LRPEP, se proponen las siguientes modificaciones y adiciones a las mismas. En la Figura 1 se muestra el mapa con la representación de las ecorregiones en los límites del territorio peruano. 


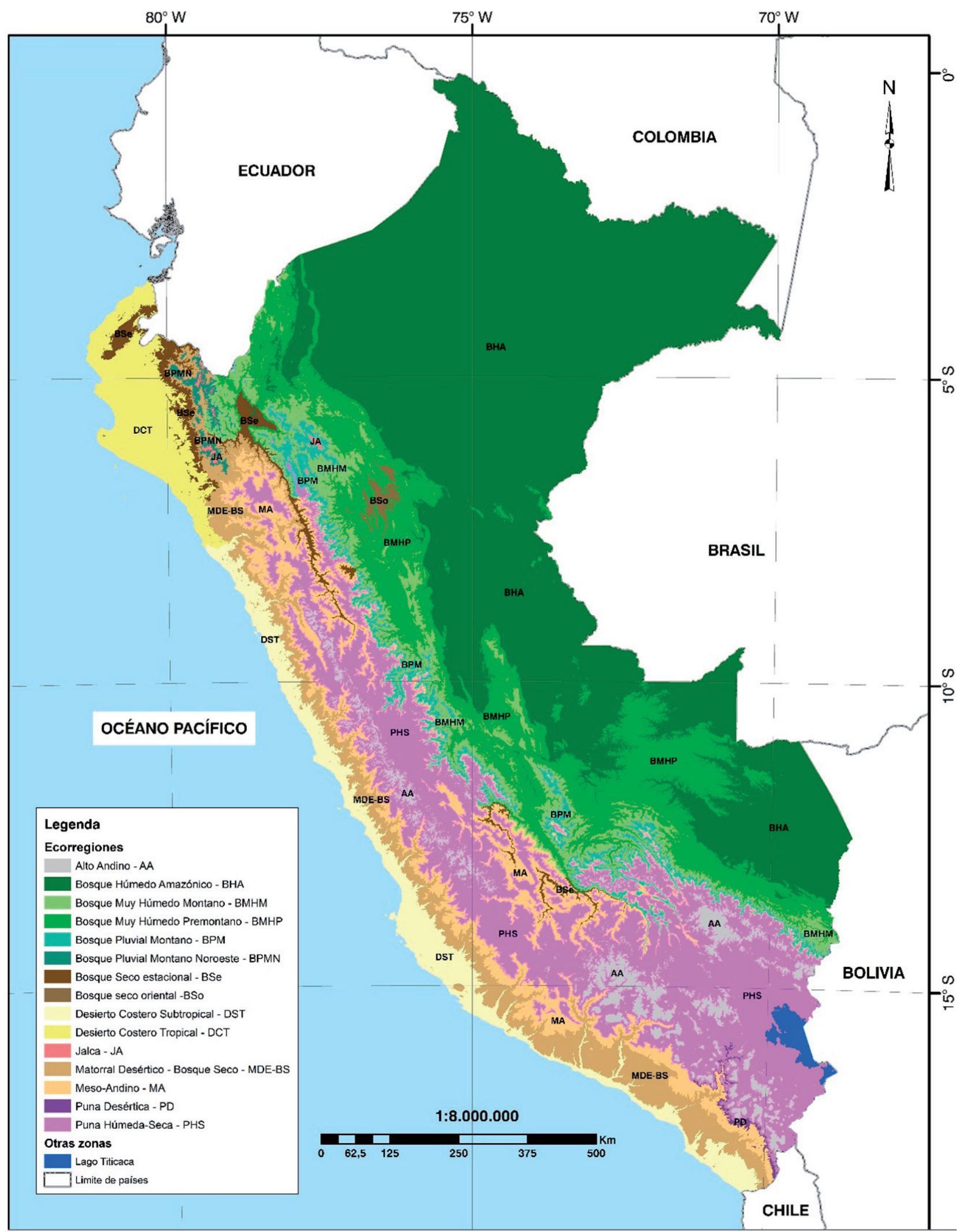

Figura 1. Mapa actualizado de ecorregiones del Perú, se muestra las 15 ecorregiones propuestas. / Updated map of ecoregions of Peru, the 15 proposed ecoregions are shown. 
- Desierto Cálido Tropical (DCT): Localizado al norte del río Chicama. Desde el nivel del mar hasta 500 (-700) m s.n.m. Involucra los departamentos de Cajamarca, La Libertad, Lambayeque, Piura y Tumbes, ocupando un área de aproximadamente 4.178.865 ha. Esta ecorregión no presenta géneros endémicos exclusivos.

- Desierto Semicálido Tropical (DST): Localizado a lo largo de la franja costera al sur del valle del Chicama. Desde el nivel del mar hasta $1.000 \mathrm{~m}$ s.n.m. Involucra los departamentos de Ancash, Arequipa, Ayacucho, Huancavelica, Ica, La Libertad, Lima, Moquegua, Tacna, ocupando un área de aproximadamente 4.819.223 ha. Esta ecorregión cuenta con un género endémico exclusivo: Diamena Ravenna, con la especie D. stenantha (Ravenna) Ravenna (Asparagaceae).

- Matorral Desértico - Bosque Seco (MDE-BS): Localizado mayormente en las laderas de la vertiente del Pacífico y ampliando su área hacia los bosques y matorrales secos del norte del país, distribuida en una franja altitudinal que va desde los $1.000(-1.600)$ hasta 2.500 m s.n.m. Involucra los departamentos de Ancash, Arequipa, Ayacucho, Cajamarca, Huancavelica, Ica, La Libertad, Lambayeque, Lima, Moquegua, Piura y Tacna, ocupando un área de aproximadamente 6.874.909 ha. Esta ecorregión cuenta con seis géneros exclusivos: Orthopterygium Hemsl., con la especie O. huaucui (A. Gray) Hemsl. (Anacardiaceae), Schistonema Schltr. con la especie $S$. weberbaueri Schltr (Asclepiadaceae), y los géneros Angeldiazia M.O. Dillon \& Zapata con A. weigendii M.O. Dillon \& Zapata, Caxamarca M.O. Dillon \& Sagást., con las especies $C$. ayabacense S. Leiva, Zapata \& M.O. Dillon y C. sanchezii M.O. Dillon \& Sagást., Nothobaccharis R.M. King \& H. Rob., con N. candolleana (Steud.) R.M. King \& H. Rob. y Syncretocarpus con las especies S. ancashino Panero \& A. Granda, S. sericeus (DC.) S.F. Blake y S. similis S.F. Blake (Asteraceae).

- Meso-Andino (MA): Se distribuye en las vertientes occidentales, las laderas de valles interandinos y el altiplano, entre 2.500 a 3.500 (-3.800) m s.n.m. Involucra los departamentos de Amazonas, Ancash, Apurímac, Arequipa, Ayacucho, Cajamarca, Cusco, Huancavelica, Huánuco, Ica, Junín, La Libertad, Lima, Madre de Dios, Moquegua, Pasco, Puno y Tacna, ocupando un área de aproximadamente 8.610.977 ha. Esta ecorregión solo cuenta con tres géneros endémicos exclusivos: Diora Ravenna, con la especie D. cajamarcaensis (Poelln.) Ravenna. Paraselinum H. Wolff, con la especie $P$. weberbauri H. Wolff (Asparagaceae), Paquirea Panero \& S.E. Freire, con la especie P. lanceolata $(H$. Beltrán \& Ferreyra) Panero \& S.E. Freire (Asteraceae).

- Puna Húmeda y Seca (PHS): Comprende la franja andina de casi todos los departamentos, entre 3.500 $(-3.800)$ y $(-4.500) 4.800 \mathrm{~m}$ s.n.m. Involucra a los departamentos de Amazonas, Ancash, Apurímac, Arequipa, Ayacucho, Cajamarca, Cusco, Huancavelica, Huánuco, Ica, Junín, La Libertad, Lima, Madre de Dios, Moquegua, Pasco, Puno, San Martín y Tacna, ocupando un área de aproximadamente 20.232.205 ha. Esta ecorregión cuenta con un género endémico exclusivo de Cactaceae: Oroya, con las especies $O$. borchersii (Bödeker) Backeberg, O. peruviana (K. Schumann) Britton \& Rose, y O. peruviana subsp. depressa (Rauh \& Backeberg) Ostolaza.

- Puna Desértica (PD): Incluye la franja andina de Moquegua, Tacna y la parte occidental de Puno, distribuyéndose entre los 3.800 y 4.200 m s.n.m. y ocupando un área de aproximadamente 199.967 ha. Esta ecorregión no presenta géneros endémicos exclusivos.

- Jalca (JA): Inicialmente se le refería como Páramo (PAR), comprende a las zonas de transición entre los Páramos del norte de Sudamérica y la Puna del centro y sur de Sudamérica, se distribuye entre (-3.200) 3.500 y $4.200 \mathrm{~m}$, de los departamentos de Amazonas, Lambayeque, Piura y parte de Cajamarca y San Martín, ocupando un área de aproximadamente 99.682 ha. Esta ecorregión no presenta géneros endémicos exclusivos.

- Alto-Andino (AA): Incluye las franjas altitudinales por encima de los (-4.500) 4.800 m s.n.m. de los departamentos de Ancash, Apurímac, Arequipa, Ayacucho, Cusco, Huánuco, Huancavelica, Ica, Junín, La Libertad, Lima, Moquegua, Pasco, Puno y Tacna, ocupando un área de aproximadamente 3.338 .853 ha. Esta ecorregión cuenta con dos géneros endémicos exclusivos de Brassicaceae: Catadysia O.E. Schulz, con la especie C. rosulans O.E. Schulz, y Dactylocardamum Al-Shehbaz, con la especie D. imbricatifolium AlShehbaz.

- Bosque Seco oriental (BSo): Comprende al bosque seco oriental ubicado en la zona del "Huallaga central" entre los ríos Huallaga y Mayo en el departamento de San Martín, altitudinalmente se distribuye entre los 100 y $500 \mathrm{~m}$ s.n.m., ocupando un área de aproximadamente 373.157 ha. Esta ecorregión no presenta géneros endémicos exclusivos.

- Bosque Seco estacional (BSe): Incluye los bosques secos ecuatoriales, entre 500 y $1.600 \mathrm{~m}$ en los departamentos de Lambayeque, Piura y Tumbes, involucrando a los bosques pertenecientes a la Reserva de Biosfera del Noroeste (Parque Nacional Cerros de Amotape, Reserva Nacional Tumbes y Coto de Caza El Angolo), los cuales se distribuyen hasta por encima de los $2.000 \mathrm{~m}$ s.n.m., y los bosques secos de los valles interandinos en los departamentos de Amazonas, Ancash, Apurímac, Ayacucho, Cajamarca, Cusco, Huánuco, Huancavelica, La Libertad, los cuales se distribuyen altitudinalmente desde los 1.000 y 2.500 m s.n.m. En total ocupa un área de aproximadamente 1.753.221 ha. Esta ecorregión 
presenta tres géneros endémicos exclusivos: Lasiocereus F. Ritter, con las especies L. fulvus F. Ritter y L. rupicola F. Ritter (Cactaceae), Rauhia Traub, con las especies $R$. decora Ravenna, R. multiflora (Kunth) Ravenna, $R$. occidentalis Ravenna y $R$. staminosa Ravenna (Amaryllidaceae), y Johnstonia Tortosa, con la especie J. axilliflora (M.C. Johnst.) Tortosa (Rhamnaceae).
- Bosques Pluviales Montanos (BPM): Corresponden a los bosques montanos de la vertiente oriental, que se distribuyen entre 2.500 y 3.500 m s.n.m. en los departamentos de Amazonas, Cusco, Huánuco, Junín, Madre de Dios, Pasco, Puno y San Martín, ocupando un área de aproximadamente 3.193.961 ha. Esta ecorregión no presenta géneros endémicos exclusivos.

TABLA 1. Lista de géneros endémicos reconocidos en el LRPEP y su respectiva actualización al año 2016. / List of endemic genera recognized in the LRPEP and their respective update to the year 2016.

\begin{tabular}{|c|c|c|c|c|c|}
\hline GÉNERO & FAMILIA & $\begin{array}{l}\text { NÚMERO DE } \\
\text { ESPECIES }\end{array}$ & $\begin{array}{l}\text { HÁBITO DE } \\
\text { CRECIMIENTO }\end{array}$ & $\begin{array}{l}\text { ECORREGIÓN SEGÚN LEÓN } \\
\text { et al. }(2007)\end{array}$ & ACtUALIZACIÓN \\
\hline Angeldiazia $^{1}$ & Asteraceae & 1 & Hierba & - & MDE-BS \\
\hline Ascidiogyne & Asteraceae & 2 & Hierba & PAR & BPM; JA; PHS \\
\hline Aynia & Asteraceae & 1 & $\begin{array}{l}\text { Hierba / } \\
\text { Subarbusto }\end{array}$ & BMHP & BMHP \\
\hline Bishopanthus & Asteraceae & 1 & Arbusto & BMHM & ВMHМ \\
\hline Calymmanthium & Cactaceae & 2 & Cactus & MDE; BS & MDE-BS; BSE \\
\hline Catadysia & Brassicaceae & 1 & Hierba & AA & $\mathrm{AA}$ \\
\hline Caxamarca $^{1}$ & Asteraceae & 2 & Hierba & - & MDE-BS \\
\hline Cephalacanthus & Acanthaceae & 1 & Subarbusto & BMHP & BMHP \\
\hline Chucoa $^{2}$ & Asteraceae & 1 & Subarbusto & MA & - \\
\hline Dactylocadamum & Brassicaceae & 1 & Hierba & AA & AA \\
\hline Diamena & Asparagaceae & 1 & Hierba & DST & DST \\
\hline Diora & Asparagaceae & 1 & Hierba & MA & MA \\
\hline Dysaster ${ }^{1}$ & Asteraceae & 1 & Hierba & - & BPMN \\
\hline Ellenbergia & Asteraceae & 1 & Hierba & BMHM & BMHM \\
\hline Filarum & Araceae & 1 & Hierba & BHA & BHA \\
\hline Guraniopsis & Cucurbitaceae & 1 & Liana $^{4}$ & BMHM & BMHM \\
\hline Hughesia & Asteraceae & 1 & Liana & ВМНM & ВМНМ \\
\hline Huthia $^{2}$ & Polemoniaceae & 2 & Arbusto & MDE; MA & - \\
\hline Inkaliabum $^{1}$ & Asteraceae & 1 & $\begin{array}{l}\text { Hierba / } \\
\text { Subarbusto }\end{array}$ & - & BMHP \\
\hline Johnstonia & Rhamnaceae & 1 & Liana & $\mathrm{BS}$ & BSE \\
\hline Laccopetalum & Ranunculaceae & 1 & Hierba & PSH; PAR; AA & PSH; JA; AA \\
\hline Lasiocereus & Cactaceae & 2 & Cactus & MDE & $\mathrm{BSE}$ \\
\hline Matucana & Cactaceae & 16 & Cactus & MDE; MA; BSE; PSH & $\begin{array}{l}\text { MDE-BS; MA; BSE; } \\
\text { PSH }\end{array}$ \\
\hline Mila & Cactaceae & 3 & Cactus & DST; MDE & DST; MDE-BS; MA \\
\hline Monocostus & Costaceae & 1 & Hierba & BHA & BHA \\
\hline Nothobaccharis & Asteraceae & 1 & Arbusto & MDE & MDE-BS \\
\hline Orophochilus & Acanthaceae & 1 & Arbusto & BMHP & BMHP \\
\hline Oroya & Cactaceae & 3 & Cactus & PSH; AA & PHS \\
\hline Orthopterygium & Anacardiaceae & 1 & Arbusto & MDE & MDE-BS \\
\hline Paquirea $^{1}$ & Asteraceae & 1 & Arbusto & - & MA \\
\hline Parasalinum & Apiaceae & 1 & Hierba & MA & MA \\
\hline
\end{tabular}




\begin{tabular}{|c|c|c|c|c|c|}
\hline GÉNERO & FAMILIA & $\begin{array}{l}\text { NÚMERO DE } \\
\text { ESPECIES }\end{array}$ & $\begin{array}{l}\text { HÁBITO DE } \\
\text { CRECIMIENTO }\end{array}$ & $\begin{array}{l}\text { ECORREGIÓN SEGÚN LEÓN } \\
\text { et al. }(2007)\end{array}$ & ACTUALIZACIÓN \\
\hline Pseudonoseris $^{2}$ & Asteraceae & 3 & $\begin{array}{l}\text { Hierba / } \\
\text { Subarbusto }\end{array}$ & MA & - \\
\hline Pygmaeocereus & Cactaceae & 3 & Cactus & DST & DST; MDE-BS \\
\hline Rauhia & Amaryllidaceae & 4 & Hierba & MDE; BS & $\mathrm{BS}$ \\
\hline Schistonema & Asclepiadaceae & 1 & Liana $^{4}$ & MA & MDE-BS \\
\hline Schizotrichia & Asteraceae & 1 & $\begin{array}{l}\text { Hierba / } \\
\text { Subarbusto }\end{array}$ & PAR; BMHM & MA; JA; BMHM \\
\hline Stilpnophyllum ${ }^{2}$ & Rubiaceae & 1 & Arbusto & BHA & - \\
\hline Syncretocarpus & Asteraceae & 3 & $\begin{array}{l}\text { Hierba / } \\
\text { Subarbusto }\end{array}$ & MDE; MA & MDE-BS \\
\hline Trichlora & Amaryllidaceae & 4 & Hierba & MA; PSH & MA; PSH \\
\hline Trichosanchezia & Acanthaceae & 1 & $\begin{array}{l}\text { Hierba / } \\
\text { Subarbusto }\end{array}$ & BHA & ВНА; ВMHР \\
\hline Ulleophytum & Asteraceae & 1 & Liana & BMHP & ВMHP \\
\hline Weberbauerella $^{2}$ & Fabaceae & 2 & Hierba & DST & - \\
\hline Weberbauerocereus ${ }^{3}$ & Cactaceae & 8 & Cactus & - & - \\
\hline
\end{tabular}

Nota: Hábito de crecimiento: se emplea las mismas descripciones usadas en el LRPEP. / Growth habit: the same descriptions used in the LRPEP

${ }^{1}$ Géneros endémicos nuevos para la flora peruana. / New endemic genera for the Peruvian flora.

${ }^{2}$ Géneros que han perdido la categoría de endémicos. /Genera that have lost the category of endemic.

${ }^{3}$ Considerado como endémico en la publicación "Todos los cactus del Perú" (Ostolaza 2014). / Considered as endemic in the publication

"Todos los cactus del Perú" (Ostolaza 2014).

${ }^{4}$ En LRPEP el hábito señalado es bejuco. /LRPEP the habit indicated is bejuco.

- Bosques Pluviales Montanos Noroeste (BPMN): Corresponde a los bosques montanos de la vertiente occidental por debajo de la ecorregión de Jalca, distribuyéndose entre los 2.500 y (3.200) 3.500 m s.n.m. en los departamentos de Cajamarca, Lambayeque y Piura, ocupando un área de aproximadamente 486.742 ha. Esta ecorregión presenta un género endémico exclusivo de Asteraceae: Dysaster H. Rob. \& V.A. Funk, con la especie Dysaster cajamarcensis H. Rob. \& V.A. Funk.

- Bosques Muy Húmedos Montanos (BMHM): Corresponde a los bosques montanos por debajo de la ecorregión de Bosques Pluviales Montanos, se distribuyen entre 1.500 y $2.500 \mathrm{~m}$ s.n.m. en los departamentos de Amazonas, Ayacucho, Cajamarca, Cusco, Huánuco, Junín, Loreto, Madre de Dios, Pasco, Puno, San Martín y Ucayali, ocupando un área de aproximadamente 6.015.010 ha. Esta ecorregión presenta cuatro géneros endémicos exclusivos: Bishopanthus H. Rob., con la especie B. soliceps H. Rob., Ellenbergia Cuatrec., con la especie E. glandulata Cuatrec., Hughesia R.M. King \& H. Rob., con la especie H. reginae R.M. King \& H. Rob. (Asteraceae), y Guraniopsis Cogn., con la especie G. longipedicellata Cogn. (Cucurbitaceae)
- Bosques Muy Húmedos Premontanos (BMHP): Comprende a los bosques premontanos de la vertiente oriental, se distribuyen entre 500 y 1.500 m s.n.m. en los departamentos de Amazonas, Apurímac, Ayacucho, Cajamarca, Cusco, Huánuco, Huancavelica, Junín, Loreto, Madre de Dios, Pasco, Puno, San Martín y Ucayali, ocupando un área aproximada de 14.894.781 ha. Esta ecorregión presenta cinco géneros endémicos exclusivos: Cephalacanthus Lindau, con la especie $C$. maculatus Lindau, Orophochilus Lindau, con la especie O. stipulaceus Lindau (Acanthaceae), Aynia H. Rob., con la especie $A$. pseudascaricida H. Rob., Inkaliabum D.G. Gut., con la especie I. diehlii (H. Rob.) D.G. Gut. y Uleophytum Hieron., U. scandens Hieron. (Asteraceae).

- Bosques Húmedos Amazónicos (BHA): Corresponde a los bosques amazónicos marcadamente no estacionales e inundables de la denominada "selva baja", se distribuyen entre los 0 y 500 m s.n.m. en los departamentos de Amazonas, Cajamarca, Cusco, Huánuco, Junín, Loreto, Madre de Dios, Pasco, Puno, San Martín y Ucayali, ocupando un área de aproximadamente 54.028.808 ha. Esta ecorregión presenta dos géneros endémicos exclusivos: Filarum Nicolson, con la especie $F$. manserichense Nicolson (Araceae), Monocostus K. Schum. con la especie M. uniflorus (Poepp. ex Petersen) Mass (Costaceae). 


\section{DISCUSIÓN}

A continuación se presenta una comparación con propuestas anteriores.

\section{LAS OCHO REgiones naturales (Pulgar-Vidal 1996)}

Pulgar-Vidal considera que una región natural es "un área continua o discontinua, en la cual son comunes o similares el mayor número de factores del medio ambiente natural (clima, relieve, suelo, subsuelo, aguas subterráneas, aguas superficiales, mar, flora, fauna, grupos humanos, latitud y altitud), y que, dentro de dichos factores, el hombre juega papel principalísimo como el más activo agente modificador de la naturaleza". Bajo este concepto, Pulgar-Vidal delimita ocho regiones naturales, las cuales explicamos de manera breve a continuación:

- Chala o Costa: Se extiende en toda la costa peruana, desde la orilla del mar hasta los 500 m s.n.m., la amplitud de esta región natural es variable, llegando a los $150 \mathrm{~km}$ en Piura, mientras que para los departamentos del sur, la franja altitudinal de $0-500 \mathrm{~m}$ s.n.m. varía de $1 \mathrm{~km}$ a menos de $40 \mathrm{~km}$, tomando de referencia la distancia de la ciudad de Tacna al mar, en línea recta. El límite altitudinal de $500 \mathrm{~m}$ s.n.m., corresponde al comienzo de la influencia de las neblinas en las estribaciones andinas de la costa.

- Yunga: Se sitúa en ambas vertientes (occidental y oriental) de la Cordillera de los Andes de Perú, debido a variaciones topográficas entre ambas vertientes, se subdivide en dos zonas, correspondiendo la Yunga fluvial a la presente en la vertiente oriental, mientras que la Yunga marítima se encuentra en la vertiente occidental. Los límites altitudinales de la Yunga fluvial son desde los 1.000 hasta los 2.300 m s.n.m., mientras que la Yunga marítima va desde los 500 hasta los 2.300 m s.n.m.

- Quechua: Se sitúa de manera continua en ambas vertientes (occidental y oriental) de la Cordillera de los Andes de Perú, distribuyéndose entre los 2.300 hasta los $3.500 \mathrm{~m}$ s.n.m. En esta región se presentan los bosques de Aliso (Alnus jorullensis Kunth), de origen natural o cultivados, principalmente en riveras de ríos y/o zonas húmedas. Presenta una topografía generalmente ondulada, que puede llegar a ser escarpada, exceptuando en la zona de los valles del río Mantaro y Urubamba.

- Suni o Jalca: Se sitúa en ambas vertientes (occidental y oriental) de la Cordillera de los Andes de Perú, distribuyéndose entre los 3.500 hasta los 4.000 m s.n.m. El relieve de la región Suni es variado, en la parte centro y sur del Perú presenta estrechas fajas inundables, también hay sectores con planos muy inclinados que se pueden aprovechar de manera reducida para la agricultura (excluyendo la manipulación antrópica). En la parte norte presenta áreas con ondulaciones suaves que permiten su uso.

- Puna: Se distribuye entre los 4.000 hasta los $4.800 \mathrm{~m}$ s.n.m., y de manera continua en ambas vertientes de la Cordillera de los Andes de Perú. A diferencia de la región Suni, la Puna es considerada de manera general como una gran llanura elevada, su presencia continua depende de la elevación de los Andes, pudiendo ubicarse a ambos lados de la cordillera, si esta sobrepasa los 5.000 m s.n.m., si es menor, la puna se presenta en el remate de las cumbres, con una topografía suave a abrupta por laderas afiladas y nacientes de quebradas.

- Janca o Cordillera: Se ubica por sobre los 4.800 m s.n.m., correspondiendo a la cima de las cadenas montañosas que conforman la Cordillera de los Andes de Perú, por lo cual no presenta una distribución continua, como sí ocurre con las otras regiones naturales. Presenta una topografía escarpada, que se alza directamente sobre la Puna, la Janca puede presentar áreas sin cubierta de nieve, la cual es denominada cordillera negra.

- RupaRupa o Selva alta: Se sitúa de manera continua en la vertiente oriental de la Cordillera de los Andes de Perú. Distribuyéndose altitudinalmente entre los 400 hasta los 1.000 m s.n.m. La región RupaRupa presenta una topografía muy compleja, donde se intercalan valles, lomadas, áreas montañosas, ladera y pongos.

- Omagua o Selva baja: También conocida como Amazonía, corresponde a toda la llanura amazónica que se ubica al este de la vertiente oriental de la Cordillera de los Andes, hasta el comienzo de la misma, 400 m s.n.m., aproximadamente.

La propuesta de Pulgar-Vidal, difiere en varios aspectos con respecto a la presente propuesta, principalmente al establecimiento de regiones naturales continuas en ambas vertientes de la Cordillera de los Andes y los valles interandinos que se encuentran en estas. La región Chala o Costa albergaría a las ecorregiones de Desierto Costero Tropical y Desierto Costero Subtropical (por debajo de los 500 m s.n.m.). La región Yunga albergaría a las ecorregiones de Desierto Costero Subtropical (por encima de los $500 \mathrm{~m}$ s.n.m.), Matorral Desértico-Bosque Seco, Bosque Seco estacional, Bosque Muy Húmedo Premontano (por encima de $\operatorname{los} 1.000$ m s.n.m.) y Bosque Muy Húmedo Montano. La región Quechua albergaría a las ecorregiones MesoAndino, Bosque Pluvial Montano, Bosque Pluvial Montano Noroeste, y la franja entre 2.300 hasta 2.500 m s.n.m., de las ecorregiones de Bosque Seco estacional y Matorral Desértico - Bosque Seco. Las regiones de Suni y Janca albergarían las ecorregiones de Jalca, Puna Desértica y Puna Húmeda-Seca. La región RupaRupa ocuparía parte de la ecorregión de Bosque Muy Húmedo Premontano, mientras que la región Omagua estaría dentro de la ecorregión de Bosque Húmedo Amazónico. 
Las regiones ecológicas del Perú (Zamora 1996)

Zamora define a las regiones ecológicas del Perú como "extensos ecosistemas implicados en un proceso dinámico de interdependencia recíproca que tiene vida propia", siendo una representación integrada de ambientes continuos o discontinuos (debido a la complejidad fisiográfica) y los factores ambientales relacionadas a estas (clima, flora y fauna típicas, condiciones litológicas y edáficas). Zamora identifica 18 regiones ecológicas, de las cuales 16 corresponden a ecosistemas terrestres. Las 16 ecorregiones se encuentran agrupadas dentro de cuatro grandes ecosistemas:

- Ecosistemas áridos y semiáridos, en donde se agrupan las regiones ecológicas de Desierto Cálido Tropical (DCT), Desierto Semicálido Tropical (DSC), Desierto Frío Altoandino (DF), Matorral Desértico (MD) y Bosques Secos (BS).

- Ecosistemas subhúmedos a húmedos mesoandinos, en donde se agrupan las regiones ecológicas de Estepa (E) y Estepa Húmeda (EH).

- Ecosistemas fríos altoandinos, en donde se agrupan las regiones ecológicas de Páramo (P), Tundra Altoandina (TA) y Nival (N).

- Ecosistemas de trópico húmedo, en donde se agrupan las regiones ecológicas de Bosque Pluvial (BP), Bosques muy Húmedos (BMH), Bosque Húmedo Tropical (BHT), Bosque Húmedo Tropical Hidromórfico (BHTh), Bosque Húmedo Tropical Estacional (BHTe) y Sabana Hidromórfica (SH).

Los ecosistemas áridos y semiáridos delimitados por Zamora guardan correlación espacial con las ecorregiones de DCT, DST, MDE-BS, BS propuestas en este trabajo, exceptuando el Desierto Frío Altoandino, cuya área se encuentra dentro de las ecorregiones de MA, PHS, PD.

Los ecosistemas subhúmedos a húmedos mesoandinos y fríos altoandinos están circunscritos a cuatro ecorregiones, Meso-Andino (MA), Puna Desértica (PD), Puna HúmedaSeca (PHS) y Alto-Andino (AA). La Estepa y Estepa Húmeda se encuentran dentro de las ecorregiones de MA y PHS, el Páramo o Puna se encuentran casi enteramente dentro de las ecorregiones de PD y PHS, por último Tundra Altoandina y Nival están dentro de la ecorregión AA.

Los ecosistemas de trópico húmedo presentan áreas similares con las ecorregiones de Bosque Húmedo Amazónico (BHA), Bosque Muy Húmedo Premontano (BMHP), Bosque Muy Húmedo Montano (BMHM), Bosque Pluvial Montano (BPM) y Bosque Pluvial Montano Noroeste (BPMN). El Bosque Húmedo Tropical, Bosque Húmedo Tropical Estacional, Bosque Húmedo Tropical Hidromórfico, parte de los Bosques muy Húmedos y la Sabana Hidromórfica, se encuentran dentro de las ecorregiones de BHA y BMHP. La parte restante de los Bosques muy Húmedos se encuentra dentro de la ecorregión de BMHM. Los Bosques Pluviales se encuentran divididos en dos ecorregiones, BPM y BPMN.
Las Ecorregiones del Perú (Brack \& Mendiola 2000)

Brack \& Mendiola definen a las ecorregiones como "un área geográfica que se caracteriza por condiciones homogéneas en lo referente a clima, suelos, hidrología, flora y fauna, y donde los diferentes factores actúan en estrecha interdependencia". Reconociendo 11 ecorregiones en el Perú, correspondiendo dos de ellas al ambiente marino (Mar Tropical, Mar frío de la corriente peruana), mientras que las nueve restantes al ambiente terrestre, de las cuales hablaremos a continuación:

- Desierto del Pacífico: Se extiende a lo largo de la costa, con un ancho variable, desde los 0 hasta los (-600, en su parte norte) $1.000 \mathrm{~m}$ s.n.m., donde termina la influencia de las neblinas invernales.

- Bosque seco ecuatorial: Los bosques tropicales secos se extienden en la faja costera de 100 a $150 \mathrm{~km}$ de ancho en los departamentos de Tumbes, Piura, Lambayeque y La Libertad y la porción seca del valle interandino del río Marañón. Ambas porciones están comunicadas a través del paso de Porculla (2.100 m s.n.m., aprox.). En el departamento de Tumbes llega hasta el nivel del mar y luego se va alejando hacia la vertiente occidental de los Andes del Perú, más de los 1.500 m s.n.m., en el valle interandino del río Marañón, ocupa las partes más bajas hasta cerca de los $2.800 \mathrm{~m}$ s.n.m.

- Bosque tropical del Pacífico: Se extiende a lo largo de la costa del Pacífico, desde el norte del Perú hasta América Central. En el Perú comprende una pequeña área en el departamento de Tumbes, en El Caucho.

- Serranía esteparia: Se extiende a lo largo del flanco occidental andino, desde el departamento de La Libertad hasta el norte de Chile, entre los 1.000 a 3.800 m s.n.m., en promedio. Los límites altitudinales superiores varían según la zona, para la zona sur es mayor, mientras que para la zona norte va disminuyendo a medida que se acerca a la línea ecuatorial.

- Puna y los altos Andes: Se extiende desde el departamento de Cajamarca, al sur del paso de Porculla, hasta Chile y Argentina, distribuyéndose altitudinalmente desde los 3.500-3.800 hasta los 5.200 m s.n.m., y desde allí hasta los $6.700 \mathrm{~m}$ s.n.m. se extienden las nieves perpetuas.

- Páramo: Se extiende desde Venezuela hasta el Perú, al norte del paso Porculla, desde los 2.100 (aprox.) hasta por encima de los (-3.200) 3.400 m s.n.m. Se distribuye en las cuencas altas de los ríos Quirós, Huancabamba y San Ignacio, en los departamentos de Cajamarca y Piura.

- Selva alta: Se extiende por todo el flanco oriental andino. En el norte del Perú penetra profundamente hacia ambos flancos del valle del Marañón y pasa a las vertientes del Pacífico en Piura, Lambayeque y Cajamarca. Se divide en tres zonas, bosques de lluvias de las vertientes occidentales (desde la cuenca del río Chicama hacia el norte), los bosques de lluvias en el valle del río Marañón, y la selva alta en las vertientes orientales. 
- Bosque tropical amazónico o selva baja: Comprende la Amazonía por debajo de los 800 m s.n.m., siendo la ecorregión más extensa del país. Esta ecorregión es parte de los bosques amazónicos que se distribuyen en Bolivia, Brasil, Colombia, Ecuador, Guayana, Venezuela y Perú.

- Sabana de palmeras: Corresponde a una formación vegetal del oriente boliviano, se presenta en el Perú solo en las pampas del río Heath, en el departamento de Madre de Dios.

La propuesta de ecorregiones del Perú de Brack \& Mendiola (2000) es una de las más empleadas actualmente, debido a que caracteriza el territorio peruano por la combinación de diferentes factores interdependientes. Es por ello que presenta una gran similitud con esta actualización de ecorregiones del Perú, definidas en primera instancia en el LRPEP.

La ecorregión de Desierto del Pacífico presenta límites altitudinales muy similares con la ecorregión de Desierto Costero Subtropical, mientras que el Desierto Costero Tropical se encontraría dentro de la ecorregión de Bosque seco ecuatorial. Las ecorregiones de Bosque Tropical del Pacífico y el Bosque Seco ecuatorial forman parte de la ecorregión de Bosque Seco estacional; cabe mencionar que el Bosque seco oriental, el cual está considerado en este trabajo, no se encuentra descrito en la propuesta de Brack y Mendiola.

La ecorregión de Serranía esteparia engloba las ecorregiones de Matorral Desértico-Bosque Seco (MDBS) y Meso-Andino (MA). Las ecorregiones de Puna $\mathrm{y}$ altos Andes se corresponden con las ecorregiones de Puna Desértica (PD), Puna Húmeda-Seca (PHS) y AltoAndino (AA). La ecorregión de Páramo contiene parte de las ecorregiones de Jalca (Ja) y parte de la ecorregión de Bosque Pluvial Montano Noroeste (BPMN), ya que solo considera los departamentos de Cajamarca y Piura, mientras en la presente propuesta, la Jalca se distribuye en los departamentos de Amazonas, Cajamarca, Lambayeque, Piura y San Martín.

La ecorregión de Selva alta reúne a varias de las ecorregiones propuestas en el presente trabajo, las cuales son: Bosque Húmedo Amazónico (por encima de los 500 m s.n.m.), Bosque Muy Húmedo Premontano (BMHP), Bosque Muy Húmedo Montano (BMHM), Bosque Pluvial Montano (BPM), Bosque Pluvial Montano Noroeste, parte del Bosque Seco estacional (BSe), y parte del Meso-Andino (MA).

Las ecorregiones de Bosque Húmedo Amazónico (por debajo de los 500 m s.n.m.) y Sabana de palmeras, forman parte de la ecorregión de Bosque Húmedo Amazónico (BHA) propuesto en este trabajo.

Ecorregiones del Libro Rojo de Plantas Endémicas del Perú (León ET AL. 2007)

En el presente artículo, se ha procurado mantener los límites espaciales y altitudinales de las ecorregiones propuestas en el LRPEP, a la vez que se proponen algunas modificaciones, con la finalidad de un mejor entendimiento de las propias ecorregiones. Al igual que propuestas anteriores, su delimitación geográfica y altitudinal, corresponde a una conjugación de factores climáticos, topográficos, de la biota presente, incorporando el conocimiento actual del desarrollo fitogeográfico de los ecosistemas que las componen.

A continuación detallamos algunos aspectos de las ecorregiones.

- Desierto Costero Tropical (DCT) y Desierto Costero Subtropical (DST): El reconocimiento de los límites altitudinales de la franja desértica de Perú ha sido relacionado directamente con el promedio de la altitud máxima en la que se desarrolla el ecosistema de lomas (Weberbauer 1945, Ferreyra 1983, Rundel et al. 1991), 1.000 m s.n.m. (Ellenberg 1959, Rundel et al. 1991). Siendo este límite altitudinal el considerado para el DST, el cual contiene a los sistemas de lomas desde el departamento de La Libertad hasta el departamento de Tacna.

La separación entre DCT y DST obedece al límite más norte del sistema de lomas, el cual corresponde a las lomas del cerro Campana en el departamento de La Libertad (Rodríguez et al. 2012). El LRPEP propone como divisoria el río Chicama, ya que se encuentra a aproximadamente $15 \mathrm{~km}$ de distancia (al norte) de las lomas del cerro Campana.

El límite altitudinal del DCT corresponde al promedio de altitud de los bosques secos costeros de los departamentos de Lambayeque, Piura y Tumbes, exceptuando los bosques secos de montaña (MINAM 2015).

- Bosque Seco estacional (BSe) y Bosque Seco oriental (BSo): Originalmente, el LRPEP solo contemplaba el sistema de bosques secos ecuatoriales y las laderas bajas de los valles secos entre (500-) 1.000 y 2.500 m s.n.m., las cuales podrían referirse a los bosques secos estacionales de los valles interandinos (que agrupan los sistemas de bosques secos estacionales del Marañón, Mantaro y Apurímac. Linares-Palomino 2006, Linares-Palomino et al. 2011), todo ello bajo la denominación de Bosque Seco, dejando de lado a los bosques secos estacionales de Tarapoto (conocido también como bosques secos estacionales orientales) (Linares-Palomino 2004, 2006, García-Villacorta 2009, Linares-Palomino et al. 2011). Cabe mencionar que en la propuesta de Zamora (1996), los bosques secos estacionales de Tarapoto son integrados en la región ecológica de Bosque Seco, dentro de la zona ecológica denominada "Bosques secos de fondos de valle del flanco oriental andino".

Los análisis de relaciones fitogeográficas entre los sistemas mencionados (Linares-Palomino et al. 2006, Linares-Palomino et al. 2011), definen como núcleos 
florísticos hermanos al sistema de bosques secos ecuatoriales y los bosques secos estacionales de los valles interandinos, los cuales corresponden a la ecorregión de BSe, separando a los bosques secos estacionales de Tarapoto, en la ecorregión de BSo.

- Matorral Desértico - Bosque Seco (MD-BS): Esta ecorregión está delimitada por la distribución altitudinal de la especie endémica Orthopterygium huaucui (Anacardiaceae), 1.200 a 2.500 (-2.800) m s.n.m. (León et al. 2013), la cual es una representante de un tipo de vegetación xerofítica que ha permanecido con pocas alteraciones en alrededor de 15 Ma (Jiménez 2013). Un patrón similar a lo propuesto por LinaresPalomino (2006) y Pennington et al. (2010), para los bosque secos estacionales de los valles interandinos, a partir del Mioceno tardío. Estudios fitosociológicos en el departamento de Arequipa, demuestran que existe una clara separación entre las comunidades vegetales xerofíticas que aparecen entre 1 os 1.100 a 2.200 m s.n.m. y las que ocurren por encima de los $2.700 \mathrm{~m}$ s.n.m. (Montesinos et al. 2015a).

- Meso-Andino (MA): La ecorregión MA comprende áreas transicionales entre la ecorregión de Puna (Húmeda-Seca o Desértica) y las ecorregiones de Matorral Desértico - Bosque Seco, en la vertiente occidental, Bosque Seco estacional, en los valles interandinos, y Bosque Muy Húmedo Montano, en la vertiente oriental. Esta ecorregión abarca una franja altitudinal que va desde los 2.500 hasta los $3.500(-3.800)$ m s.n.m.

Dentro de esta ecorregión podemos encontrar a los bosques nublados secos, los cuales se distribuyen desde el $7^{\circ} \mathrm{S}$ hasta $13.3^{\circ} \mathrm{S}\left(-16^{\circ} \mathrm{S}\right.$, Koepcke 1961), $\mathrm{y}$ entre los 2.600 hasta los $3.200 \mathrm{~m}$ s.n.m. (Valencia 1992), presentándose de manera más discontinua y fragmentada en dirección norte a sur. Koepcke (1961), postuló que estos bosques dispersos son relictos de una banda boscosa continua que existió cuando anteriormente el clima era más húmedo. Hacia el sur, los bosques nublados son reemplazados por matorralespajonales, con una estructura similar a la prepuna del norte de Chile (Latorre et al. 2003, Squeo et al. 2006), la cual se distribuye altitudinalmente entre los 2.700 hasta 3.500 m s.n.m. Montesinos et al. (2012) explican que este tipo de vegetación se amplía hasta cerca de los 3.800 m s.n.m. en el departamento de Moquegua. Los límites altitudinales entre la ecorregión Meso-Andina y La Puna Desértica en el departamento de Moquegua es reafirmada por Montesinos et al. (2015b) a través de estudios fitosociológicos de la vegetación de puna de ese departamento.

En la vertiente oriental, la ecorregión Meso-Andina se presenta de manera similar a la prepuna boliviana (López 2000), especialmente en la parte alta de los valles de los ríos Apurímac, San Lorenzo y Santo Tomás en los departamentos de Apurímac y Cusco (obs. pers.).

- Puna Desértica (PD): La ecorregión de PD, está relacionada directamente con la distribución de la especie de ave Rhea pennata (d'Orbigny, 1834), también conocido como "Suri", el cual se encuentra principalmente en la franja de (-3.500) 3.800 a 4.200 (-4.300) m s.n.m. en los departamentos de Moquegua, Tacna y parte occidental del departamento de Puno (Villanueva 2006, Lleellish et al. 2007). Además que el límite inferior de esta ecorregión (3.800 m s.n.m.) concuerda con el límite superior de la distribución altitudinal de los matorrales altoandinos, los cuales forman parte de la comunidad de plantas de prepuna (ecorregión Meso-Andina), identificados por Montesinos et al. (2012, 2015b), en el departamento de Moquegua.

- Puna Húmeda-Seca (PHS): Los bofedales (también llamados "occonales") corresponden a una unidad de vegetación donde predominan especies de plantas adaptadas a zonas con alto grado de saturación de agua (Tovar 1973). De manera general, las familias predominantes a nivel de cobertura vegetal son Asteraceae, Juncaceae, Plantaginaceae y Poaceae (Valencia et al. 2013). La disponibilidad de agua puede ser permanente o temporal y se les encuentra en los alrededores de ríos, lagunas, zonas cercanas a deshielos, ojos de agua (puntos de surgencia de agua dulce, o áreas en contacto con la napa freática). Se distribuyen altitudinalmente, en promedio, entre los $3.500(-3.800) \mathrm{y}$ (-4.500) 4.800 m s.n.m., ocasionalmente hasta altitudes de $5.200 \mathrm{~m}$ s.n.m. (obs. pers.), y geográficamente en casi todos los departamentos del Perú (exceptuando Amazonas, Lambayeque, Loreto, Madre de Dios, Piura, Tumbes y Ucayali) (MINAM 2015). En el rango altitudinal en que se presentan, los bofedales se forman bajo condiciones ambientales con fuertes variaciones de temperatura diaria, radiaciones UV intensas, fuertes vientos e hipoxia (Valencia et al. 2013).

Los bofedales juegan un rol muy importante para el sustento (alimentación, anidamiento, entre otros servicios) de diferentes especies de fauna poco común y/o endémica de los Andes (Villagrán \& Castro 1997). De igual manera, tienen un valor muy importante para los pobladores andinos, a los que proveen diferentes servicios ecosistémicos, como el aprovisionamiento de agua dulce y alimentación del ganado ovino, vacuno y de camélidos altoandinos, en especial en la temporada seca, donde su uso se intensifica (Browman 1984, Tapia \& Flores 1984).

Por lo expuesto, se considera que los bofedales son una unidad de vegetación representativa en la Cordillera de los Andes, por lo que se considera que los límites altitudinales y geográficos de la ecorregión de PHS coinciden con los de esta unidad de vegetación.

- Jalca (JA): La definición de Páramo y Jalca en Perú, o 
los límites geográficos de los mismos han sido temas de larga discusión en los últimos 20 años. El término "Jalca" ha sido empleado por diferentes autores (Weberbauer 1945, Sánchez-Vega 1976, 1997, Simpson, 1983, Becker 1988, Llatas-Quiroz \& López-Mesones 2005, SánchezVega \& Dillon 2006, Chocce et al. 2010, Galán de Mera et al. 2015), de manera exclusiva, para describir a la vegetación por encima de los $3.000(-3.200,3.500) \mathrm{m}$ s.n.m., en los departamentos de Amazonas, Cajamarca, Lambayeque y Piura, en Perú. Mientras que Monasterio (1980) y Luteyn (1999), incluyen a la Jalca dentro de los Páramos que se extienden en los Andes del norte de Sudamérica, siendo de igual manera empleado en el LRPEP (León et al. 2007).

Sin embargo, de manera general se describe a los Páramos como los pajonales por encima de los 3.200 m s.n.m. (aproximadamente) y que están caracterizados por la presencia de los frailejones (subtribu Espeletiinae Cuatrec., Asteraceae) (Diazgranados 2012a). Lo cual está sustentado por análisis filogeográficos, los cuales concluyen que el origen de esta subtribu monofilética fue en Venezuela (en sus Páramos) y tuvo una rápida (y reciente) radiación en los Andes del norte, hace unos 2-4 millones de años (Diazgranados 2012a, Diazgranados \& Barber 2017), lo cual concuerda con la estimación de la edad y desarrollo de los Páramos (Van der Hammen \& Cleef 1986, Hooghiemstra \& Van der Hammen 2004). La mayor riqueza de especies de la subtribu Espeletiinae se encuentra en Colombia y Venezuela, reportándose solo una especie en Ecuador, la cual se distribuye hasta la sierra de Llanganates (Diazgranados 2012a, 2012b). La Jalca ha sido reconocida anteriormente como una unidad biogeográfica independiente de los Páramos (Sánchez-Vega 1976, Simpson 1983) o como parte de otras categorías biogeográficas independientes, exclusivas al territorio peruano (Britto \& Arana 2014). Estos planteamientos son consistentes con las observaciones realizadas por Weigend (2002, 2004) en la zona de Amotape-Huancabamba (en la que se distribuye la Jalca), donde se sustenta que esta zona debe considerarse como un área fitogeográfica única, debido a las características compuestas de su flora residente, al poseer elementos florísticos del norte y sur de los Andes (Weigend, 2002).

Con base a lo señalado en los párrafos anteriores, se considera adecuado emplear la definición de Jalca (Sánchez-Vega \& Dillon 2006) para esta ecorregión.

- Alto Andino (AA): El LRPEP propuso como límite inferior de esta ecorregión los $4.200 \mathrm{~m}$ s.n.m. Sin embargo, considerando que se ha redefinido el límite de la ecorregión de Puna Húmeda-Seca y que la vegetación predominante a partir de altitudes mayores a 4.500 está asociada a suelos crioturbados (Cano et al. 2010, 2011) (obs. pers. en los departamentos de Ancash, Apurímac,
Arequipa, Cusco, Huancavelica, Lima, Moquegua), por lo que se ha modificado el límite inferior de esta ecorregión a $4.500(-4.800)$.

- Bosque Húmedo Amazónico (BHA) y Bosque Muy Húmedo Premontano (BMHP): Los bosques amazónicos de Perú que se encuentran entre los 0 a 1.500 m s.n.m., han sido separados en base a sus características climáticas (Young \& León 2001). Los bosques amazónicos entre 0 a 500 m s.n.m., denominados mayormente como "selva baja" o "bosques tropicales y subtropicales de tierras bajas" (Young 1992), pertenecen a la ecorregión de BHA. Mientras que los bosques amazónicos entre 500 a 1.500 m s.n.m., son denominados mayormente como "premontanos" (Young 1992, Young \& León 2001), los cuales pertenecen a la ecorregión de BMHP.

- Bosque Muy Húmedo Montano (BMHM), Bosque Pluvial Montano (BPM) y Bosque Pluvial Montano Noroeste (BPMN): Young \& Valencia (1992), definen al "bosque montano" como la vegetación boscosa ubicada por encima de los 1.000 m s.n.m. en Perú, tanto en la vertiente occidental y oriental de los Andes. Así mismo, separan los bosques montanos en continuos (los pertenecientes a la vertiente oriental y parte norte de la vertiente occidental, ubicados en los departamentos de Cajamarca, Lambayeque y Piura) y fragmentados (en el resto de departamentos). Con base en características climáticas, los "bosques nublados" (otra denominación de los "bosques montanos"), comienzan a partir de los 500 m s.n.m. (Young \& León 2001). Mientras que la franja altitudinal entre los 1.500 a 3.500 m s.n.m. albergan a los bosques montanos húmedos, propiamente dichos (Young 1992, León et al. 1992, Young \& León 1999, Young \& León 2001, Antón \& Reynel 2004).

Los bosques montanos húmedos del Perú son separados en sus vertientes, esto es sustentado por diversos análisis biogeográficos realizados a nivel regional y global (Cabrera \& Willink 1973, Morrone 2001, Olson et al. 2001), y local (Dillon et al. 1995, Weigend 2002, 2004, Weigend et al. 2005, Britto \& Arana 2014). Los bosques montanos húmedos del noroeste de Perú corresponden a la ecorregión de BPMN.

Los bosques montanos húmedos de la vertiente oriental son separados por sus características fisionómicas, estructurales, riqueza de especies, composición de especies y endemismos (Gentry 1992, Young 1992, León et al. 1992, Young \& León 1999, Van der Werff \& Consiglio 2004, Britto \& Arana 2014).

Los bosques montanos húmedos bajos, que se distribuyen aproximadamente entre los 1.500 a 2.500 m s.n.m. (Young \& León 1992, Young \& León 1999), presentan mayor altura de dosel arbóreo, mayor riqueza de especies de árboles y de especies de plantas leñosas, en comparación con los bosques montanos altos, que van de los 2.500 a 3.500 m s.n.m., aproximadamente. 
Estos bosques montanos húmedos bajos corresponden a la ecorregión de Bosque Muy Húmedo Montano (BMHM).

Los bosques montanos altos presentan menores valores con respecto al dosel arbóreo y riqueza de especies leñosas (Gentry 1992, Young \& León 1999), pero presentan una mayor riqueza de endemismos en sus bosques (Van der Werff \& Consiglio 2004, Britto \& Arana 2014), lo cual se debe a que son áreas transicionales entre la vegetación compuesta por elementos andinos y amazónicos (Pintaud et al. 2008). Estos bosques corresponden a la ecorregión de Bosque Pluvial Montano (BPM).

Finalmente, es necesario recalcar que los límites (altitudinales y geográficos) de las ecorregiones presentadas en esta publicación corresponden a una aproximación de lo que se observa en campo, ya que la distribución espacial de las ecorregiones no se presenta de manera horizontal y continua (Fig. 2), debido a la complejidad geográfica ejercida por la cordillera de los Andes en el territorio peruano, la cual ha influenciado en los patrones de diversidad de distintos grupos de plantas que conforman la flora peruana (Mutke et al. 2014).

\section{AGRADECIMIENTOS}

A los miembros de la RSAS por sus opiniones y sugerencias para los manuscritos, a los revisores del presente trabajo por las sugerencias bibliográficas para ampliar la discusión de resultados, y en especial al equipo de Ambiente y Geociencias de SNC-Lavalin Perú, por hacerme partícipe en sus proyectos en todo el país, los cuales me han permitido tener una visión panorámica de la amplia riqueza de los componentes de la biodiversidad en Perú.

\section{REFERENCIAS}

Andersson, L. 1995. Tribes and genera of the Cinchoneae complex (Rubiaceae). Annals of the Missouri Botanical Garden 82: 409-427.

Andersson, L., Taylor, C.M. 1994. Rubiaceae-CinchoneaeCoptosapelteae. In: Harling, G.W., Andersson, L. (eds.), Flora of Ecuador, pp. 78-81. University of Göteborg. Göteborg.

Antón, D., Reynel, C. 2004. Relictos de bosques de excepcional diversidad en los Andes centrales del Perú. Herbario de

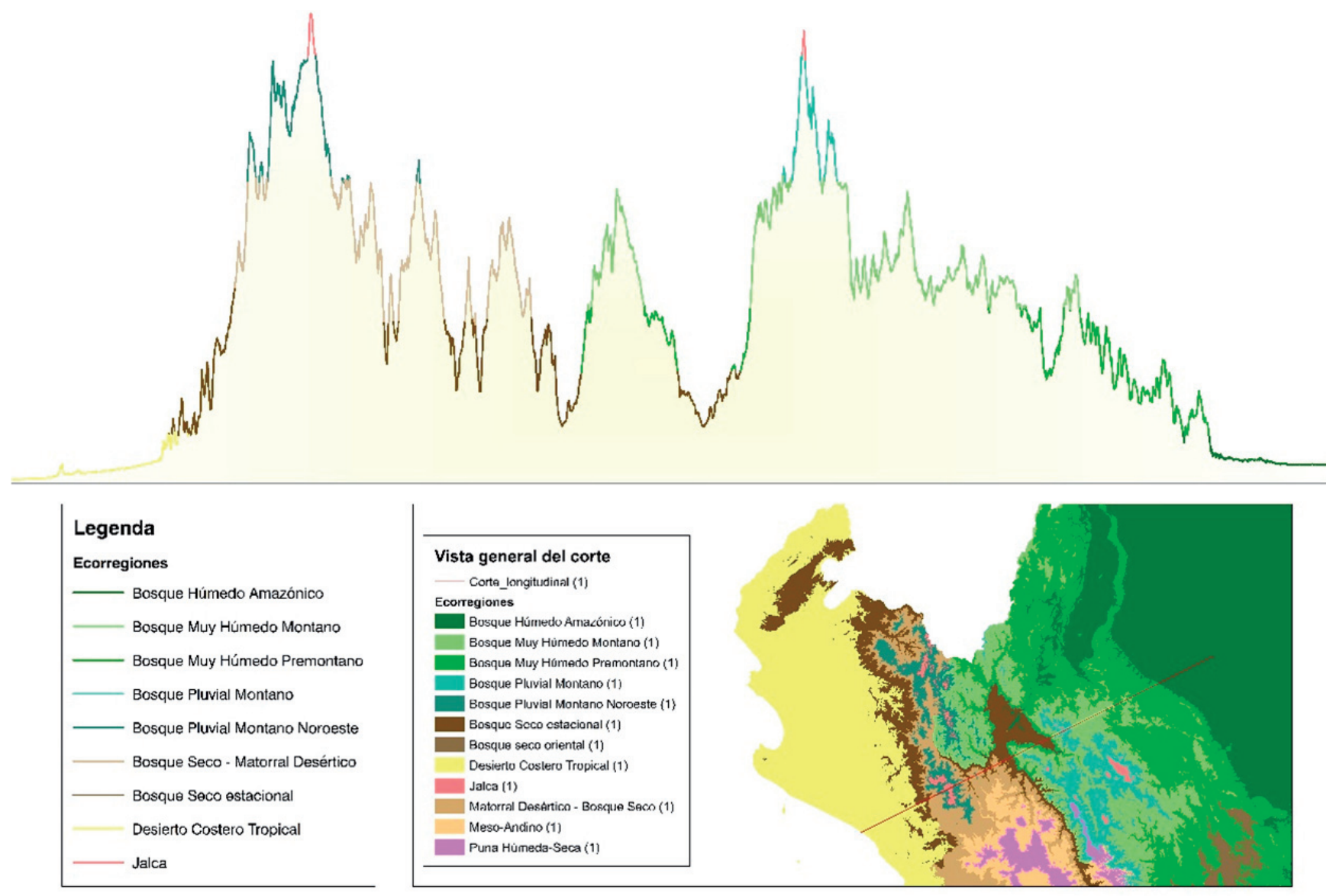

FiguRa 2. Sección longitudinal de las ecorregiones en el norte de Perú con base en la complejidad topográfica del territorio peruano. / Longitudinal section of the ecoregions in the north of Peru based on the topographic complexity of the Peruvian territory. 
la Facultad de Ciencias Forestales, Universidad Nacional Agraria La Molina. Lima, Perú. 313 pp.

Becker, B. 1988. Degradation and rehabilitation of Andean ecosystems-an example from Cajamarca. Angewandte Botanik 62(3-4): 147-160.

Brack, A., Mendiola, C. 2000. Ecología del Perú. Parte II. Las regiones naturales del Perú. Bruño, Lima, Perú. 169 pp.

Brako, L., Zarucchi, J. 1993. Catalogue of the Flowering Plants and Gymnosperms in Peru. Monograph in Systematic Botany from the Missouri Botanical Garden 45. $1286 \mathrm{pp}$.

Britto, B., Arana, C. 2014. Corotipos preliminares de Perú en base a la distribución de la familia Asteraceae. Darwiniana nueva serie 2(1): 39-56.

Browman, D. 1984. Pastoralism and development in the Andes. Journal of Arid Environments 7: 313-318.

Cabrera, A.L., Willink, A. 1973. Biogeografía de América Latina. Serie de Biología, Monografía $\mathrm{n}^{\circ}$ 13. Washington D.C. Secretaría General de la Organización de los Estados Americanos. 120 pp.

Cano, A., Mendoza, W., Castillo, S., Morales, M., La Torre, M.I., Aponte, H., Delgado, A., Valencia, N., Vega, N. 2010. Flora y vegetación de suelos crioturbados y hábitats asociados en la cordillera Blanca, Áncash, Perú. Revista Peruana de Biología 17(1): 95-103.

Cano, A., Delgado, A., Mendoza, W., Trinidad, H., Gonzáles, P., La Torre, M.I., Chanco, M., Roque, J., Valencia, N., Navarro, E. 2011. Flora y vegetación de suelos crioturbados y hábitats asociados en los alrededores del abra Apacheta, Ayacucho-Huancavelica (Perú). Revista Peruana de Biología 18(2): 169-78.

Chocce, M., Dalström, S., Hágsater, E., Arnaiz, J. 2010. Epidendrum jalcaënse (Orchidaceae), a new species from northern Peru. Lankesteriana 9(3): 529-531.

Consortium for Spatial InFormation (CGIAR-CSI). SRTM 90m Digital Elevation Database v4.1. http://srtm.csi.cgiar.org/ Vista: Agosto 15, 2015.

Diazgranados, M. 2012a. Phylogenetic and biogeographic relationships within the Espeletiinae (family Asteraceae), an endemic subtribe of the South American Páramos. PhD Thesis. Doctoral Dissertation. Saint Louis University, USA. 233 pp.

Diazgranados, M. 2012b. A nomenclator for the frailejones (Espeletiinae Cuatrec., Asteraceae). PhytoKeys 16: 1-52.

Diazgranados, M., Barber, J. 2017. Geography shapes the phylogeny of frailejones (Espeletiinae Cuatrec., Asteraceae): a remarkable example of recent rapid radiation in sky islands. PeerJ 5:e2968. DOI 10.7717/ peerj. 2968

Dillon, M.O., Sagástegui A., A., Sánchez V., I., Llatas, S. Hensold, N. 1995. Floristic inventory and biogeographic analysis of montane forests in northern Peru. In: Churchill, S.P., Balslev, H., Forero, E., Luteyn, J.L. (eds.), Biodiversity and Conservation of Neotropical Montane Forests, pp. 251-270. The New York Botanical Garden Press, Bronx.

Dillon, M.O., ZAPATA, M. 2010. Angeldiazia weigendii (Asteraceae, Senecioneae), a new genus and species from northern Peru. Arnaldoa 17(1): 19-24.

EllenberG, H. 1959. Über den Wasserhaushalt tropischer Nebeloasen in der Küstenwüste Perus. Berichte der
Deutschen Botanischen Gesellschaft 73: 449-462.

Ferreyra, R. 1983. Los tipos de vegetación de la costa peruana. Anales del Jardín Botánico de Madrid 40: 241-256.

FunK, V., Robinson, H., TANGerini, A. 2013. Whatever happened to Bishopanthus (Compositae, Liabeae)? Phytokeys 30: 65-73.

Galán de Mera, A., Sánchez-Vega, I., Montoya, J., Linares, E., CAmpos, J., Vicente, J.A. 2015. La vegetación del norte del Perú: de los bosques a la Jalca en Cajamarca. Acta Botanica Malacitana, 40: 157-190.

García-Villacorta, R. 2009. Diversidad, composición y estructura de un hábitat altamente amenazado: los bosques estacionalmente secos de Tarapoto, Perú. Revista Peruana de Biología 16(1): 081-092.

Gentry, A. 1992. Diversity and floristic composition of Andean forests of Peru and adjacent countries: implications for their conservation. In: Young, K.R., Valencia, N. (eds.), Biogeografía, ecología y conservación del bosque montano en el Perú, pp. 11-29. Memorias del Museo de Historia Natural "Javier Prado" 21, Universidad Nacional Mayor de San Marcos, Lima, Perú.

Gutierrez, D.G. 2010. Inkaliabum, a new Andean genus of Liabeae (Asteraceae) from Peru. Boletin de la Sociedad Argentina de Botánica 45 (3-4): 363-372.

Hooghiemstra, H., Van der Hammen, T. 2004. Quaternary IceAge dynamics in the Colombian Andes: developing an understanding of our legacy. Philosophical transactions of the Royal Society of London Series B, Biological sciences 359: 173-180

Huber, O., Rirna, R. 1997. Peru. En: Huber, O., Riina, R. (eds.), Glosario Fitoecológico de las Américas. Vol. 1. América del Sur: países hispanohablantes. Caracas, Venezuela. 416421 pp., UNESCO.

JiMÉNEZ, V. 2013. Diversidad genética y relaciones filogenéticas de Orthopterygium huaucui (A. Gray) Hemsley, una Anacardiaceae endémica de la vertiente occidental de la Cordillera de los Andes. Tesis. Título Profesional de Biólogo con mención en Botánica. Facultad de Ciencias Biológicas. Universidad Nacional Mayor de San Marcos, Lima, Perú. 107 pp.

KoEpCKe, H.-W. 1961. Synokologische Studien an der Westseite der peruvianischen Anden. Bonner Zoologischer Abhandlungen 29: 1-320.

Latorre, C., Betancourt, J.L., Rylander, K.A., Quade, J., Matthei, O. 2003. A vegetation history from the arid prepuna of northern Chile $\left(22-23^{\circ} \mathrm{S}\right)$ over the last 13,500 years. Palaeogeography, Palaeoclimatology, Palaeoecology 194: 223-246.

Leiva, S., Zapata, M. 2008. Caxamarca ayabacense (Asteraceae: Senecioneae) una nueva especie del Norte del Perú. Arnaldoa 15(2): 177-183.

León, B., Roque, J., Ulloa, C., Jørgensen, P.M., Pitman, N., Cano, A. 2007. Libro Rojo de las Plantas endémicas del Perú (diciembre 2006). Revista Peruana de Biología. Edición Especial 13(2): $971 \mathrm{pp.}$

León, B., Young, K., Brako, L. 1992. Análisis de la composición florística del bosque montano oriental del Perú. En: K.R. Young \& N. Valencia (eds.), Biogeografía, ecología y conservación del bosque montano en el Perú, pp. 141 154. Memorias del Museo de Historia Natural "Javier 
Prado" 21, Universidad Nacional Mayor de San Marcos, Lima, Perú.

León, B., Young, K.R., Roque, J. 2013. Evaluación del estado poblacional del huaucui y de las comunidades vegetales que integra: Bases para una estrategia de conservación en la vertiente occidental peruana. Informe final del convenio de donación $\mathrm{N}^{\circ} 70$-2009-APECO-CI. 24 pp.

LinARES-PALOMINO, R. 2004. Los bosques tropicales estacionalmente secos II: Fitogeografía y composición florística. Arnaldoa 11(1): 103-138.

Linares-Palomino, R. 2006. Phytogeography and Floristics of Seasonally Dry Tropical Forests in Peru. In: Pennington, R.T., Lewis, G.P., Ratter, J.A. (eds.), Neotropical Savannas and Seasonally Dry Forests: Plant Diversity, Biogeography, and Conservation, pp. 257-280. The Systematics Association Special Volume Series 69, Taylor and Francis Group.

Linares-Palomino, R., Oliveira-Filho, A.T., Pennington, R.T. 2011. Neotropical Seasonally Dry Forests: Diversity, Endemism and Biogeography of Woody Plants. In: Dirzo, R., Mooney, H., Ceballos, G., Young, H. (eds.), Seasonally Dry Tropical Forests: Ecology and Conservation, pp. 3-21. Island Press. Washington, USA.

Llatas-Quiroz, S., López-Mesones, M. 2005. Bosques montanosrelictos en Kañaris (Lambayeque, Perú). Revista Peruana de Biología 12(2): 299-308.

Lleellish, M., Salinas, L., Chipana, E. 2007. Situación del Suri Pterocnemia pennata en el Perú. INRENA - Intendencia Forestal y de Fauna Silvestre - DCB, Lima, Perú. 12 pp. URL: http://museohn.unmsm.edu.pe/doc/suri_sit peru2007.pdf

LóPEz, R.P. 2000. La prepuna boliviana. Ecología en Bolivia 34: 45-65.

LuteYn, J.L. 1999. Páramos: a checklist of plant diversity, geographical distribution, and botanical literature. New York Botanical Garden Press, Bronx, New York, USA. $278 \mathrm{pp}$.

MINAM. 2015. Mapa nacional de cobertura vegetal: memoria descriptiva. Ministerio del Ambiente, Dirección General de Evaluación, Valoración y Financiamiento del Patrimonio Natural. Lima, Perú. 108 pp.

Monasterio, M. 1980. Estudios ecológicos en los páramos andinos. Ediciones Universidad de los Andes, Mérida. 312 pp.

Montesinos, D., Cleef, A.M., SkÝora, K.V. 2012. Andean shrublands of Moquegua, South Peru: Prepuna plant communities. Phytocoenologia 42(1-2): 29-55.

Montesinos, D., Skýora, K.V., Quipuscon-Silvestre, V., Cleef, A.M. 2015a. Species composition and phytosociology of xerophytic plant communities after extreme rainfall in South Peru. Phytocoenologia 45(3): 203-250.

Montesinos-Tubée, D., Cleef, A.M., SÝkora, K.V. 2015b. The Puna vegetation of Moquegua, South Peru: Chasmophytes, grasslands and Puya raimondii stands. Phytocoenologia 45(4): 365-397.

Morrone, J.J. 2001. Biogeografía de América Latina y el Caribe. Manuales \& Tesis SEA 3. Zaragoza, España. 148 pp.

Mutke, J., Jacobs, R., Meyers, K., Henning, T., Weigend, M. 2014. Diversity patterns of selected Andean plant groups correspond to topography and habitat dynamics, not orogeny. Frontiers of Genetics 5: 351.
Olson, D.M., Dinerstein, E., Wikramanayake, E.D., Burgess, N.D., Powell, G.V.N., Underwood, E.C., D’Amico, J.A., Strand, H.E., Morrison, J.C., Loucks, C.J., Allnutt, T.F., LAMOREuX, J.F., RicketTs, T.H., ItouA, I., WetTENGeL, W.W., Kura, Y., Hedao, P., Kassem, K. 2001. Terrestrial eco-regions of the world: A new map of life on Earth. BioScience 51(11): 933-938.

ONERN. 1976. Mapa ecológico del Perú: Guía explicativa. Oficina Nacional de Evaluación de Recursos Naturales. Lima, Perú. 146 pp.

Ostolaza, C. 2014. Todos los Cactus del Perú. MINAM. Lima, Perú. 538 pp.

Panero, J.L. 2009. Chucoa ilicifolia, a spiny Onoseris (Asteraceae, Mutisioideae: Onoserideae). Phytologia 91: 537-541.

Panero, J.L., Freire, S.E. 2013. Paquirea, a new Andean genus for Chucoa lanceolata (Asteraceae, Mutisioideae, Onoserideae). Phytoneuron 11: 1-5.

Pennington, R.T., Lavin, M., Särkinen, T., Lewis, G.P., Klitgaard, B.B., Hughes, C.E. 2010. Contrasting plant diversification histories within the Andean biodiversity hotspot. Proceedings of the National Academy of Sciences 107(31): $13783-13787$.

Pintaud, J.C., Galeano, G., Balslev, H., Bernal, R., Borchsenius, F., Ferreira, E., De Granville, J. J., Mejía, K., Millán, B., Moraes, M., Noblick, L., Stauffer, F.W., Kahn, F. 2008. Las palmeras de América del Sur: diversidad, distribución e historia evolutiva. Revista Peruana de Biología 15: 7-30.

Porter, J.M., Prather, L.A. 2008. Cantua dendritica (Polemoniaceae), a new species from Peru, and two new Cantua Names. Aliso 25(1): 31-35.

Pruski, J.F. 2012. Studies of Neotropical Compositae-VIII. The new combination Pseudonoseris glandulosa and revision of Pseudonoseris (Liabeae). Phytoneuron 113: 1-10.

Pulgar-Vidal, J. 1996. Geografía del Perú. Las ocho regiones naturales. La Regionalización Transversal. La Sabiduría Ecológica Tradicional, 10ma edición. PEISA, Lima, Perú. $218 \mathrm{pp}$

QuisPe, N.R., FuENTES, A.F. 2010. Weberbauerocereus madidiensis (Cactaceae), una Nueva Especie del Norte de Bolivia. Novon 20: 325-328.

Robinson, H., Funk, V. 2014. Dysaster cajamarcensis, a new shrubby genus and species of Astereae (Asteraceae) from Peru. Phytokeys 36: 35-40.

Rodríguez, E.R., Liza, V.E., Martínez, B.M., Pollack, L., Leiva, S., Zapata, M., Mora, M. 2012. Loma el Cerro Campana, patrimonio natural y cultural de la región La Libertad. Pueblo Continente 23(2): 330-336.

Rundel, P.W., Dillon, M.O., Palma, B., Mooney, H.A., Gulmon, S.L., Ehleringer, J.R. 1991. The phytogeography and ecology of the coastal Atacama and Peruvian deserts. Aliso 13: 1-49.

SAlDIVIA, P., FAÚNDEZ, L. 2014. Weberbauerella chilensis (Fabaceae: Papilionoideae), a new species from the Atacama Desert, Chile. Phytotaxa 156(1): 41-46.

SÁnchez-VegA, I. 1976. Contribución al estudio ecológico de la "Jalca" en el Departamento de Cajamarca-Perú. Tesis Doctoral. Universidad Nacional de Trujillo, Trujillo. 29 pp.

SÁnchez-Vega, I. 1997. Aspectos florísticos de la jalca y alternativas de manejo sustentable. Arnaldoa 4(2): 25-62.

SÁnchez-Vega, I., Dillon, M.O. 2006. Jalcas. En: Moraes, M., 
Øllgaard, B., Kvist, L.P., Borchsenius, F., Balslev, H. (eds.), Botánica Económica de los Andes Centrales, pp: 77-90. Universidad Mayor de San Andrés. La Paz, Bolivia.

SimPSON, B. 1983. An historical phytogeography of the high Andean flora. Revista Chilena de Historia Natural 56: 109-122.

Squeo, F., Cepeda, J., Olivares, N.C., Arroyo, M.T.K. 2006. Interacciones ecológicas en la alta montaña del Valle del Elqui. En: Cepeda, J. (ed.), Geoecología de los Andes desérticos. La alta montaña del Valle del Elqui, pp. 69-103. Universidad de La Serena, La Serena, Chile.

TAPIA, M., Flores, J. 1984. Pastoreo y pastizales de los Andes del sur del Perú. INIPA. Editorial Adolfo Artela. Lima, Perú. $312 \mathrm{pp}$.

Tovar, O. 1973. Comunidades vegetales de la Reserva Nacional de Vicuñas de Pampas de Galeras, Ayacucho, Perú. Publicaciones del Museo de Historia Natural Javier Prado, serie $\mathrm{B}$, Botany 27: 1-32.

Tropicos ${ }^{\circledR}$, botanical information system at the Missouri Botanical Garden. [Permanentemente actualizado] URL: http:// www.tropicos.org, Vista: Enero-Julio, 2016.

Valencia, N. 1992. Los bosques nublados secos de la vertiente occidental de los Andes del Perú. En: Young, K.R., Valencia, N. (eds.), Biogeografía, ecología y conservación del bosque montano en el Perú, pp. 155-170. Memorias del Museo de Historia Natural "Javier Prado" 21, Universidad Nacional Mayor de San Marcos, Lima, Perú.

Valencia, N., Cano, A., Delgado, A., Trinidad, H., Gonzáles, P. 2013. Composición y cobertura de la vegetación de bofedales en un macrotransecto Este-Oeste en los Andes centrales del Perú. En: Alonso, A., Dalmmeier, F., Servat, G.P. (eds.), Monitoreo de biodiversidad: Lecciones de un megaproyecto transandino, pp. 278-293. Smithsonian Institution Scholarly Press. Washington, D.C.

Van der Hammen, T., \& Cleef, A.M. 1986. Development of the high Andean páramo flora and vegetation. In: Vuilleumier, F., Monasterio, M. (eds.), High Altitude Tropical Biogeography, pp. 153-201. Oxford University Press, New York, USA.

Van der Werff, H., Consiglio, T. 2004. Distribution and conservation significance of endemic species of flowering plants in Peru. Biodiversity and Conservation 13: 16991713.

Villagrán, C., Castro, V. 1997. Etnobotánica y manejo ganadero de las vegas, bofedales y quebradas en el Loa superior, Andes de Antofagasta, Segunda Región, Chile. Chungará
(Chile) 29: 275-304.

Weberbauer, A. 1945. El mundo vegetal de los Andes Peruanos (Estudio fitogeográfico). Ministerio de Agricultura, Lima, Perú. $776 \mathrm{pp}$.

WeIGEND, M. 2002. Observations on the biogeography of the Amotape-Huancabamba zone in Northern Peru. The Botanical Review 68(1): 38-54

WeIgend, M. 2004. Additional observations on the biogeography of the Amotape-Huancabamba zone in Northern Peru: Defining the South-Eastern limits. Revista Peruana de Biología 11(2): 127-134.

Weigend, M., Rodríguez, E.F., Arana, C. 2005. The relict forests of northwest Peru and Southwest Ecuador. Revista Peruana de Biología 12(2): 185-194.

Young, K.R. 1992. Biogeography of the montane forest zone of the eastern slopes of Peru. In: Young, K.R., Valencia, N. (eds.), Biogeografía, ecología y conservación del bosque montano en el Perú, pp. 119-140. Memorias del Museo de Historia Natural "Javier Prado" 21, Universidad Nacional Mayor de San Marcos, Lima, Perú.

Young, K.R., Valencia, N. 1992. Los bosques montanos del Perú. En: Young, K.R., Valencia, N. (eds.), Biogeografía, ecología y conservación del bosque montano en el Perú, pp. 5-9. Memorias del Museo de Historia Natural "Javier Prado" 21, Universidad Nacional Mayor de San Marcos, Lima, Perú.

Young, K.R., LeÓN, B. 1992. Análisis de la composición florística del bosque montano oriental del Perú. En: Young, K.R., Valencia, N. (eds.), Biogeografía, ecología y conservación del bosque montano en el Perú, pp. 141-154. Memorias del Museo de Historia Natural "Javier Prado" 21, Universidad Nacional Mayor de San Marcos, Lima, Perú.

Young, K.R., León, B. 1999. Peru's Humid Eastern Montane Forests: An Overview of Their Physical Settings, Biological Diversity, Human Use and Settlement, and Conservation Needs. DIVA Technical Report 5. Kalø, Denmark: Centre for Research on Cultural and Biological Diversity of Andean Rainforests (DIVA). 96 pp.

Young, K.R., León, B. 2001. Perú. En: Kappelle, M., Brown, A. (eds.), Bosques Nublados del Neotrópico, pp. 549-580. Editorial IMBIO, Costa Rica.

ZAmorA, C. 1996. Las regiones ecológicas del Perú. En: Rodríguez, L.O. (ed.), Diversidad Biológica del Perú: Zonas Prioritarias para su Conservación, pp. 137-141. FANPE, GTZ-INRENA. Lima, Perú. 